\title{
Chemical Immunotherapy
}

National Cancer Institute

\section{Source}

National Cancer Institute. Chemical Immunotherapy. NCI Thesaurus. Code C15918.

The use of chemicals to enhance the immune system in treatment of cancer 